\title{
Mapping unit-cell thickness variations in thin films by post-deposition reflection high-energy electron diffraction
}

\author{
A. E. M. Smink $\odot,{ }^{1,2, *}$ Y. A. Birkhölzer $\odot,{ }^{1}$ J. van Dam, ${ }^{1}$ F. J. G. Roesthuis $\odot,{ }^{1}$ G. Rijnders, ${ }^{1}$ H. Hilgenkamp, ${ }^{1}$ and G. Koster ${ }^{1}$ \\ ${ }^{1}$ MESA+ Institute for Nanotechnology, University of Twente, P.O. Box 217, 7500 AE Enschede, The Netherlands \\ ${ }^{2}$ Max Planck Institute for Solid State Research, Heisenbergstraße 1, 70569 Stuttgart, Germany
}

(Received 26 May 2020; accepted 28 July 2020; published 20 August 2020)

\begin{abstract}
Reflection high-energy electron diffraction is a widely used tool to study the growth dynamics of thin films, in situ and operando. Here it is applied after deposition, revealing that the morphological state of the surface is connected to the amount of material underneath. We focus on the growth of $\mathrm{LaAlO}_{3}$ thin films on a $\mathrm{SrTiO}_{3}$ substrate by pulsed laser deposition, utilizing a geometrically anisotropic plasma plume. After deposition, oscillations in the intensity and width of the specular spot as a function of position are observed along the short axis of the plasma plume. Each complete oscillation represents a unit-cell difference in film thickness, as confirmed by local x-ray reflectivity measurements. We apply this approach to demonstrate the critical thickness for conductivity at the $\mathrm{LaAlO}_{3} / \mathrm{SrTiO}_{3}$ interface on a single sample. Our results introduce a straightforward method to engineer and study thickness-dependent properties of epitaxial thin films on a highly detailed level.
\end{abstract}

DOI: 10.1103/PhysRevMaterials.4.083806

\section{INTRODUCTION}

The physical properties of thin films are often strongly dependent on the thickness of the film. In some systems, this dependence is critical - especially in the material class of complex oxides, a thickness difference of a single monolayer (or unit cell, u.c.) can completely change the physical behavior of the film. For example, the emergence of ferroelectricity [1], (ferro)magnetism [2], (semi)metallic behavior [3,4], and superconductivity [5] depend critically on the film thickness in many materials. Moreover, emergent properties at oxide interfaces [6] are similarly affected by the thickness of the constituent layers: the conductivity at the $\mathrm{LaAlO}_{3} / \mathrm{SrTiO}_{3}$ interface $[7,8]$, superconductivity in the $\mathrm{FeSe} / \mathrm{SrTiO}_{3}$ system [9], and ferromagnetism at $\mathrm{LaMnO}_{3} / \mathrm{SrTiO}_{3}$ interfaces [10] are well-established examples.

To study such phenomena, it is essential to control the film thickness on the atomic scale. Developed to monitor the growth dynamics of semiconductor stacks [11-14], timeresolved reflection high-energy electron diffraction (RHEED) is very well suited to do so. As illustrated in Fig. 1, a highenergy beam of electrons hits the substrate surface under grazing incidence, where it is reflected and diffracted in a characteristic pattern. RHEED is a highly surface-sensitive, in situ probe for the surface crystal structure and morphology. During layer-by-layer growth, the formation of a new layer

\footnotetext{
*s.smink@fkf.mpg.de
}

Published by the American Physical Society under the terms of the Creative Commons Attribution 4.0 International license. Further distribution of this work must maintain attribution to the author(s) and the published article's title, journal citation, and DOI. Open access publication funded by the Max Planck Society. is characterized by a full cycle of layer coverage, leading to oscillations of the peak intensity [11] and width [12] of the spots in the diffraction pattern. Monitoring these RHEED oscillations operando, i.e., during film growth, enables the control of layer thickness on the unit-cell level.

Here, we report the observation of similar oscillations in the RHEED pattern, after the deposition of $\mathrm{LaAlO}_{3}$ thin films on a $\mathrm{SrTiO}_{3}$ substrate, as a function of position on the sample. These films are grown by pulsed laser deposition, using a vertically elongated ablation spot. The resulting plasma plume is highly confined (widespread) along the vertical (horizontal) direction, yielding a particle flux gradient away from the plume center. This flux gradient is reflected in the postdeposition observation of position-dependent RHEED oscillations. The oscillations emerge first from a certain distance away from the plume center during deposition; the connection between the particle flux gradient and the oscillation pattern is established further by a correlation between the lateral oscillation period and nominal film thickness. Comparing the lateral oscillation profiles to local x-ray reflectivity measurements shows that each full oscillation along the sample surface represents a thickness difference of exactly one unit cell. We apply this approach to deposit a $\mathrm{LaAlO}_{3}$ film with a thickness gradient from four to three unit cells, yielding a metal-insulator transition at the $\mathrm{LaAlO}_{3} / \mathrm{SrTiO}_{3}$ interface. The introduced RHEED-based method is a straightforward means to analyze local thickness variations of epitaxially grown thin films, empowering the study and engineering of thickness-dependent properties in thin films in a single growth step.

\section{METHODS}

Thin films of $\mathrm{LaAlO}_{3}$ were grown by pulsed laser deposition (PLD) on $5 \times 5 \times 0.5 \mathrm{~mm}^{3} \mathrm{SrTiO}_{3}$ substrates, using a 


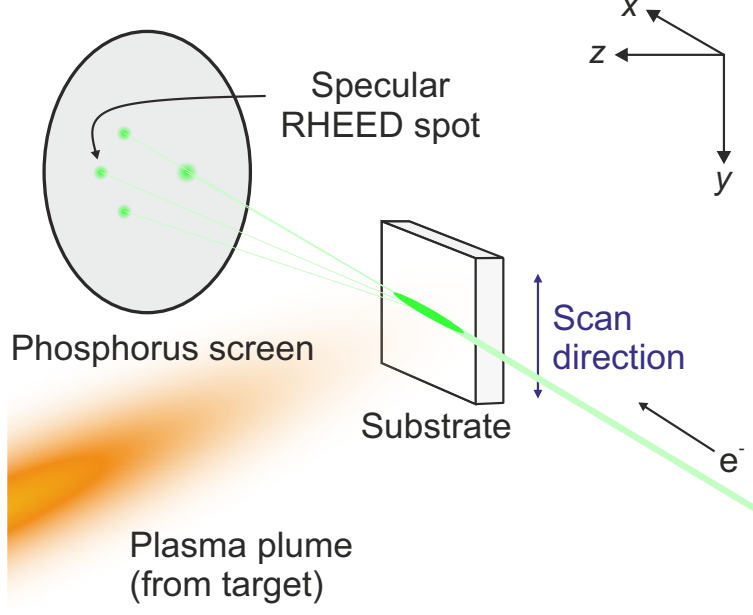

FIG. 1. Schematic of a PLD-RHEED setup. The $100-\mu$ m-wide RHEED beam (green) hits the substrate at grazing incidence, creating a diffraction pattern which is made visible by a phosphorus screen. Ideally, the center of the plasma plume hits the substrate in exactly the same position. The blue arrow indicates the scan direction to obtain lateral RHEED profiles.

single-crystalline $\mathrm{LaAlO}_{3}$ target (both provided by CrysTec $\mathrm{GmbH}$ ). The $\mathrm{O}_{2}$ process pressure was $1 \times 10^{-4}$ mbar and the substrate temperature was $850^{\circ} \mathrm{C}$, provided by a resistive heater element to which the substrate was glued by silver paint. Laser light from a KrF laser (Plasma Physik LPX 210i, wavelength $248 \mathrm{~nm}$ ) was led into the deposition chamber through an optical path, in which the beam was shaped by a stainless steel mask. This results in a rectangular spot on the target, with an area of $1.76 \mathrm{~mm}^{2}$ and a 4.8:1 elongation along the vertical direction. As illustrated in Figs. 2(a) and 2(b), the combination of the chosen laser spot and the relatively low deposition pressure leads to a widely spread plasma plume along the $x$ direction, and a fairly confined plume along the $y$ direction. Compared to studies with a more isotropically shaped plume $[15,16]$, this should lead to a large particle flux gradient along $y$, and a smaller difference in deposited material along $x$. The laser fluence was $1.3 \mathrm{~J} \mathrm{~cm}^{-2}$, the pulse frequency was $1 \mathrm{~Hz}$, and the substrate-target distance was $60 \mathrm{~mm}$. After deposition, the samples were cooled down to room temperature at a rate of $10^{\circ} \mathrm{C} / \mathrm{min}$ at deposition pressure, unless mentioned otherwise.

The RHEED setup consisted of a $35-\mathrm{kV}$ electron source [17] and a phosphorus screen, monitored by a camera. As illustrated in Fig. 1, the 100- $\mu$ m-wide RHEED beam impinges on the sample surface at grazing incidence, probing a wide area on the sample surface along the propagation direction. Prior to growth, the RHEED specular spot intensity was maximized as a function of the angles of incidence and azimuth. This approximates the optimal in-phase Bragg diffraction conditions for the substrate, where the reflections from each layer contributing to the reflected signal interfere constructively. We find that in these conditions, the amplitude of the growth oscillations is maximized. The thus obtained RHEED patterns were analyzed using the KSA400 software, and all measurements of peak intensity and the full width at half maximum (FWHM) of the beam were done on the cross-section of the specular spot along the [001] direction. Position-dependent measurements were done by moving the substrate surface along the given axis through the fixed electron beam, using a constant-speed $(0.25 \mathrm{~mm} / \mathrm{s})$ stepper motor. Because of the elongated shape of the RHEED spot on the sample surface, the RHEED pattern is independent of lateral position $x$; therefore, the sample was rotated by $90^{\circ}$ for measurements along the long axis of the plume (denoted $x$ in the figures below). Unless stated otherwise, all positiondependent measurements were done in growth conditions.

X-ray reflectivity (XRR) measurements were carried out using a Bruker D8 Discover diffractometer with a highbrilliance microfocus $\mathrm{Cu}$ rotating anode generator $(2.5 \mathrm{~kW})$, hybrid Montel optics (parallel-focusing), 0.1-mm divergence slit, a 0.3 -mm-diameter pinhole beam collimator, and an EIGER2 R $500 \mathrm{~K}$ area detector. No monochromator was used to maximize the flux from the microfocus laboratory source, sacrificing beam divergence for dynamic range, here more than seven decades, enabling XRR data collection over a wide angular range (up to $10^{\circ}$ in the $2 \theta$ direction). To collect the specular reflection data, the detector was operated in conventional zero-dimensional (OD) mode with a very small region of interest ( 33 pixels, pixel size $75 \times 75 \mu \mathrm{m}^{2}$ ) in a coupled $2 \theta-\omega$ scan. No secondary optics in the diffracted beam paths between sample and detector were installed. Faster scan modes that would exploit the full two-dimensional (2D) capabilities of the large detector, e.g., in rocking curve mode, were avoided to minimize the effect of diffuse (air) scattering.

Two-point resistance measurements versus temperature were carried out in a Quantum Design Physical Property Measurement System (PPMS), using the built-in electronics and a $5 \mu \mathrm{A}$ excitation current maximum, in two separate cooldowns of the sample.

\section{RESULTS}

\section{A. Lateral RHEED profiles}

On sample A, 10 u.c. of $\mathrm{LaAlO}_{3}$ were deposited in the standard alignment: the RHEED beam and the center of the plasma plume both pointed to the center of the substrate. The film thickness was controlled by monitoring the RHEED specular spot intensity during growth [18]. Before and after deposition, the intensity and FWHM of the specular spot were measured as a function of the vertical lateral position $y$. The results are presented in Figs. 2(c) to 2(e). Figure 2(c) shows the measurement along the $y$ axis of the bare substrate-the profile along $x$ is very similar (not shown). In principle, the substrate has a homogeneous surface and therefore, the specular spot intensity and width should be constant as a function of position. The measurement deviates from this ideal profile due to three aspects of the experimental setup: (i) a slight misalignment of the scan direction with the $x y$ plane of the substrate surface results in a monotonically changing effective cross section of the beam with the sample surface, leading to a constant slope in the reflected beam intensity; (ii) vibrations in the setup are visible as small random variations; (iii) interference and refocusing effects on the sample edges $(y= \pm 2.5 \mathrm{~mm})$ cause local peak sharpening or broadening, occasionally leading to a high maximum intensity and 
(a)

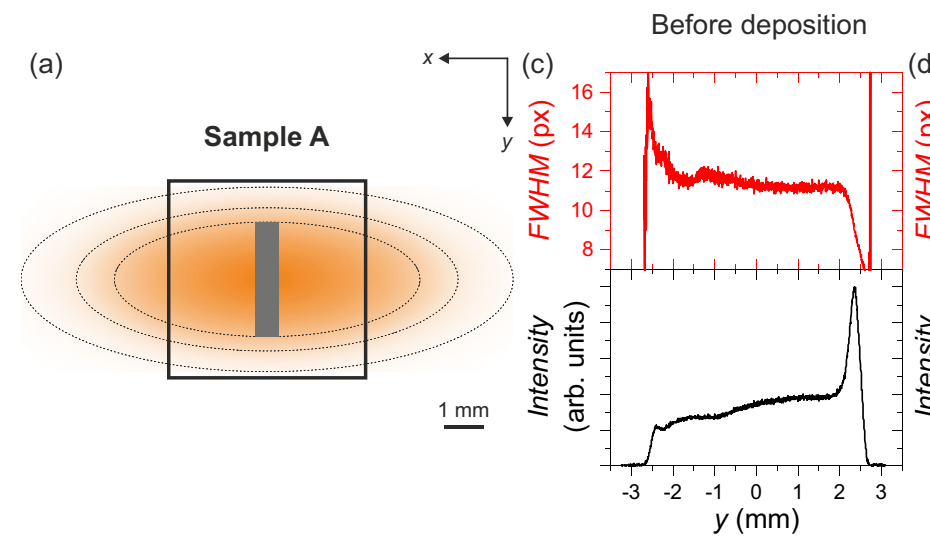

Before deposition

$1 \mathrm{~mm}$

(b)

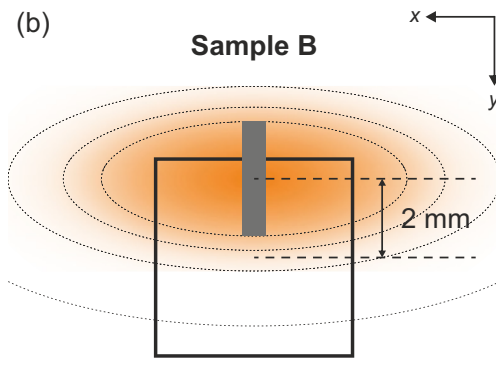

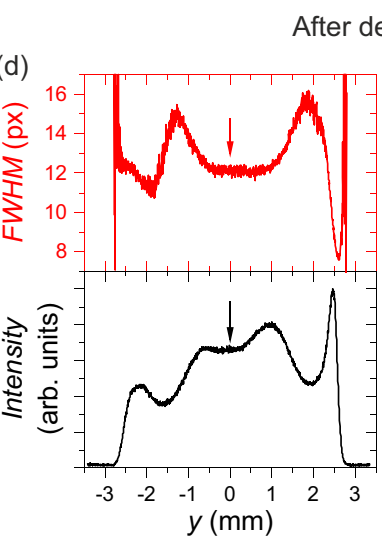

After deposition
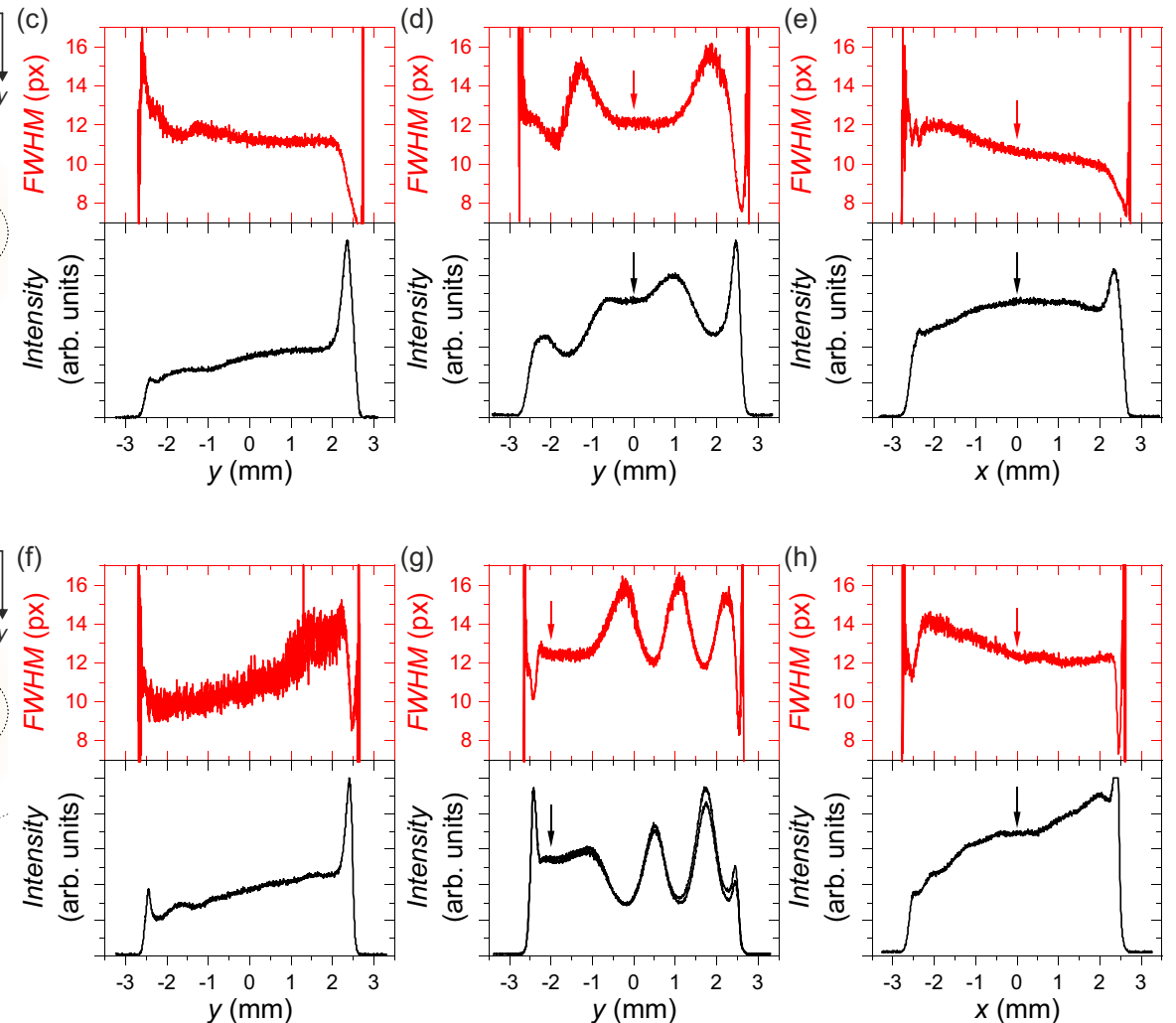

(h)

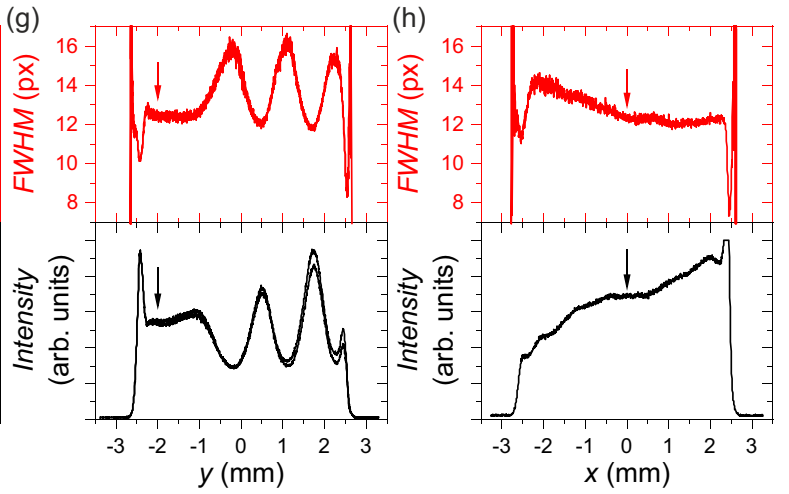

FIG. 2. Lateral RHEED profiles. (a, b) Front views of the substrate during deposition, overlaid with a schematic cross section of the plasma plume (orange) and the ablation spot on the target (gray). The dotted lines schematically indicate contours of equal particle flux. (a) On sample A, the plume and substrate centers are aligned, yielding a large homogeneous area in the middle of the sample. (b) On sample B, the plume and the substrate center are offset along $y$ (the short axis of the plume) by $2 \mathrm{~mm}$. (c) Full width at half maximum (FWHM, top panel) and maximum intensity (bottom panel) of the RHEED specular spot as a function of position $y$ on sample A, taken just before deposition. $y=0$ mm defines the center of the sample and the sample edges are at $\pm 2.5 \mathrm{~mm}$. Off the sample, the intensity drops to zero and the FWHM becomes undefined. The origins for the deviation from an ideal, flat profile as a function of position are described in the main text. [(d), (e)] Lateral RHEED profiles taken directly after the deposition of 10 u.c. $\mathrm{LaAlO}_{3}$ on sample A, along the $y$ and $x$ directions. The estimated position of the plume center during deposition is indicated by the arrows. (f)-(h) Same as (c)-(e), for sample B. These curves are taken for both scan directions, showing perfect overlap.

accompanying deviations in the FWHM signal. As will become clear in the following, these artifacts do not impede the analysis of post-deposition RHEED profiles.

After the deposition of 10 u.c. of $\mathrm{LaAlO}_{3}$, Fig. 2(d) shows a clear pattern of peaks and dips in the FWHM and intensity signals along $y$. This pattern is symmetric around $y=0.3 \mathrm{~mm}$, aside from the still present constant background slope in the intensity signal. Between $y=-0.5$ and $1.1 \mathrm{~mm}$, the FWHM signal is flat; the intensity signal shows a small dip, which we attribute to stopping the deposition slightly late [18]. Along $x$, the long axis of the plume, such a pattern is absent, as shown in Fig. 2(e). This is also the case for a control sample grown with a circular ablation spot [18], leading to a widespread plume along both $x$ and $y$ and hence, position-independent RHEED signals for both directions. We therefore conclude that the pattern of peaks and dips is related to the more confined shape of the plume in the $y$ direction.

This is even more pronounced on sample B, which was grown in the same conditions but with one difference: the plume center (and the RHEED beam) had a vertical offset of $2 \mathrm{~mm}$ with respect to the substrate center, as illustrated in Fig. 2(b). As readily observed in Fig. 2(g), the pattern of peaks and dips along $y$ now forms clear oscillations. These lateral oscillations appear only at $y>-1 \mathrm{~mm}$, below which the signal is independent of $y$, like the region on the center of sample A. The positions of the flat regions correlate with the position of the plume center, indicating that the lateral oscillations emerge first from a certain distance away from the plume center.

During the deposition of a third sample $\mathrm{C}$, the plume center was even further away from the substrate center: In fact, it was deliberately placed off the substrate at $y=-4 \mathrm{~mm}$. For monitoring purposes, the RHEED beam remained fixed at $y=-2 \mathrm{~mm}$ during growth. Figure 3(a) shows the lateral intensity profiles along $y$ for all three samples. Sample $C$ has no flat area anymore, but displays an oscillation pattern with an apparently constant period. A closer look at the oscillation pattern of sample $B$ reveals that the oscillations have a longer period closer to the plume center; further away, the period decreases to the one observed for sample $\mathrm{C}$.

On sample $\mathrm{C}$, the number of pulses required to grow 1 u.c. was larger than for all other samples studied: 17.3 instead of 


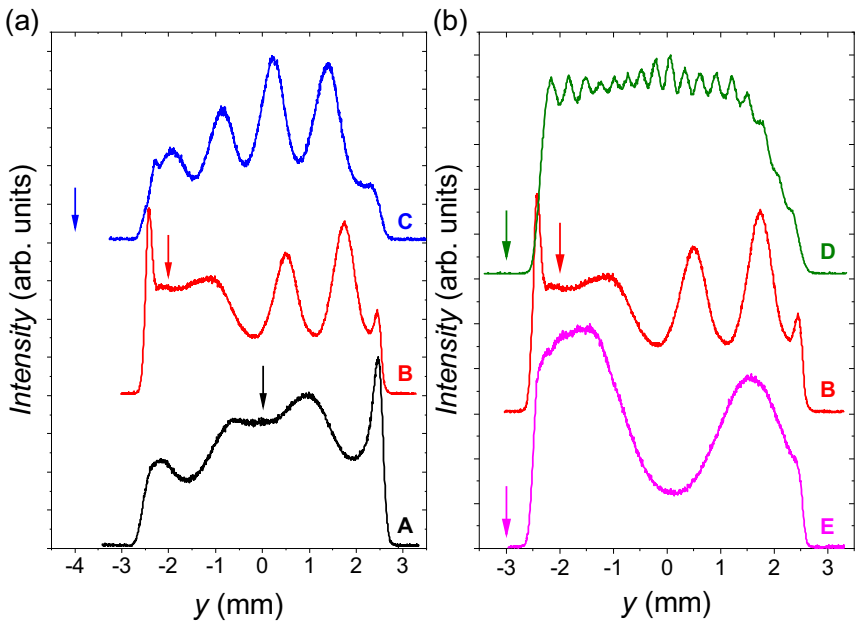

FIG. 3. Lateral RHEED intensity profiles along $y$ for varying deposition conditions. (a) On samples A through C, 10 u.c. of $\mathrm{LaAlO}_{3}$ were deposited with different offsets of the plume center to the substrate center, as indicated by the arrows. Data are offset for clarity. (b) On samples D and E, the plume center was kept at $y=$ $-3 \mathrm{~mm}$ during deposition, while growing 40 and 4 u.c. of $\mathrm{LaAlO}_{3}$ at $y=-2 \mathrm{~mm}$, respectively. The data for sample $\mathrm{B}(t=10 \mathrm{u}$.c.) is included for completeness. Like in (a), the arrows indicate position of the plume center during deposition and data are offset for clarity.

$16 \pm 0.5$ pls/u.c. We attribute this to the fact that the RHEED beam was located $2 \mathrm{~mm}$ below the plume center during deposition, which according to the schematic in Fig. 2(b) should lead to less material being deposited there. In other words, the deposition rate in our samples decreases with distance to the plume center and must thus be considered as a local property due to the particle flux gradient illustrated in Fig. 2(a).

To investigate the effect of film thickness in more detail, two more films were grown: Samples D and E had local $\mathrm{LaAlO}_{3}$ thicknesses, $t$, of 4 and 40 u.c., respectively, as measured during growth at $y=-2 \mathrm{~mm}$. The plume center was at $y=-3 \mathrm{~mm}$ during these two depositions. The resulting intensity profiles are presented in Fig. 3(b), which includes the data for sample B ( $t=10$ u.c.) for completeness. Both samples show lateral oscillations along $y$ : for sample D, 15 full oscillations are observed, whereas sample E shows one oscillation. We note that, again, no oscillations are observed along $x$ for either sample (not shown). On sample D, the period of the oscillations is constant over the whole sample; moreover, it is about four times smaller than that of sample $\mathrm{C}$ and $\mathrm{B}$, on which the $\mathrm{LaAlO}_{3}$ film is four times thinner.

Based on this correlation of the oscillation period and the film thickness, the observation of a locally varying deposition rate and the coincidence of the plume center and the region without lateral oscillations, we propose that the lateral RHEED oscillations represent a systematic thickness variation in the grown film. Such a thickness gradient would manifest itself at the film surface as a continuously changing top-layer coverage; if the thickness gradient exceeds one unit cell, the coverage would be oscillating along the sample surface. A full oscillation of coverage is reflected one-to-one in the specular spot intensity [11] and width [12]. During the layer-by-layer growth of thin films, this is observed at a fixed location as a function of deposition time; in our post-deposition measurements, as a function of position on the sample surface. This proposed scenario implies that each lateral oscillation represents a full $\mathrm{LaAlO}_{3}$ coverage cycle; hence, a thickness difference of exactly 1 u.c.

\section{B. X-ray reflectivity}

To test this hypothesis, we performed grazing-incidence XRR measurements as a function of position on samples $\mathrm{C}$ and D. Individual scans were performed perpendicular to the presumed thickness gradient (in the same direction as the RHEED beam), thus in narrow regions of approximately constant thickness. The resulting XRR data for sample $\mathrm{D}$, with varying position $y$, are presented in Fig. 4(b); the results for sample C are discussed in the Supplemental Material [18]. All the data show clear reflectivity oscillations, indicating low surface and interface roughness, as well as a high thickness homogeneity along the beam direction, in accordance with the RHEED data.

To extract information on the local film thickness, mass density, and layer roughness, these XRR data were analyzed by simulations and fitting [18]. For both samples, the mass density and surface roughness do not vary significantly with position, with values of 6.0 to $6.2 \mathrm{~g} \mathrm{~cm}^{-3}$ and 0.35 to $0.4 \mathrm{~nm}$, respectively. We note that we did not make use of a knifeedge collimator [19], which could have further increased the accuracy of these values. Contrary to the density and roughness, the extracted $\mathrm{LaAlO}_{3}$ layer thickness decreases systematically: from 15.31 to $9.84 \mathrm{~nm}$ on sample D. This corresponds to a thickness gradient from 41 to 26 u.c., a difference of 15 u.c.

In Fig. 4(c), we plot the thickness extracted from the XRR fits of both samples $C$ and $D$, versus position. The open symbols represent the peak positions of the RHEED profiles, extracted as described in the Supplemental Material [18], with a difference of 1 u.c. in the film thickness between each peak. For this dataset, the thickness at $y=-2 \mathrm{~mm}$ is determined by the RHEED oscillations during growth: 10 and 40 unit cells for sample $C$ and $D$, respectively. The very good overlap of both independently acquired datasets confirms that the post-deposition RHEED oscillations originate from a thickness gradient. In other words, RHEED can be used as a sensitive local probe to map thickness variations in epitaxially grown thin films. The resolution for such measurements is much smaller than one unit cell in thickness, as the maxima and minima of the oscillations can be identified with great precision; laterally, as indicated by the clear oscillation pattern on sample D [Fig. 3(b)], the resolution is about $50-100 \mu \mathrm{m}$. Moreover, the time required to measure a RHEED profile in our setup is less than 1 minute.

\section{Gradient across a critical thickness}

By carefully adjusting the offset between the plume and the substrate, a thickness difference of one unit cell across the surface of a sample can be engineered. This should allow the study of critical-thickness phenomena on a single sample, in a single deposition step. For the $\mathrm{LaAlO}_{3} / \mathrm{SrTiO}_{3}$ interface, a metal-insulator transition (MIT) occurs across a thickness of 
(a)

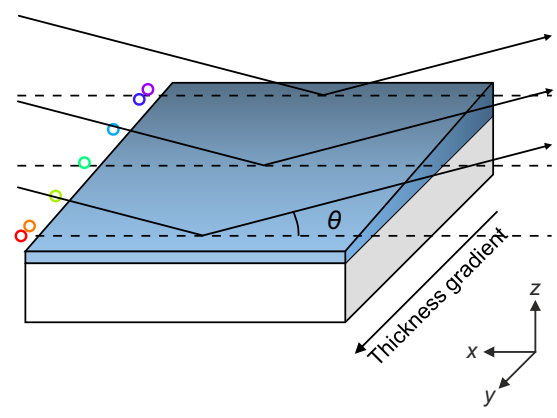

(b)

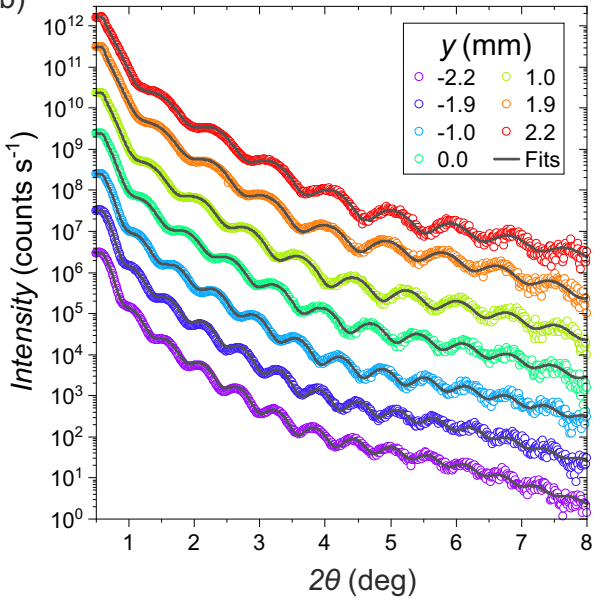

(c)

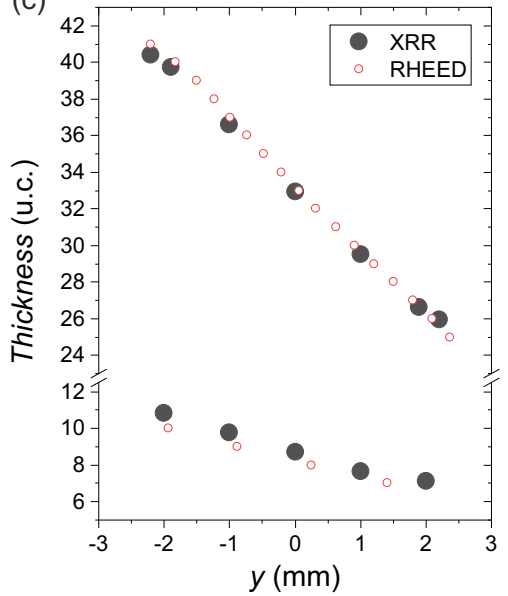

FIG. 4. Local x-ray reflectivity (XRR) measurements. (a) Measurement schematic on different positions on the sample, indicated by the colored circles, a 0.3 -mm-wide $\mathrm{x}$-ray beam is reflected at a grazing incidence $\theta$. Thickness (gradient) not to scale. (b) XRR intensity versus $2 \theta$ for varying positions on sample D. Curves are offset for clarity and solid lines are fits to the data. (c) Thickness gradients on sample C (bottom) and D (top), extracted from XRR and RHEED data.

three to four unit cells [7]. This is ascribed to an electronic reconstruction [20], which is likely triggered by the spontaneous formation of oxygen vacancies at the $\mathrm{LaAlO}_{3}$ surface as an electric potential builds with increasing film thickness [21,22].

On sample $\mathrm{F}$, we engineered a thickness gradient from four to three unit cells. Figure 5(a) shows one full oscillation in the RHEED intensity from $y \approx-1$ to $y \approx 1.7 \mathrm{~mm}$, hence a thickness variation of one unit cell. To measure the effect of this thickness gradient on interface conductivity, we prepared a dedicated sample G: This sample was structured using an $\mathrm{AlO}_{x}$ hard mask and subsequent lithographic patterning and wet etching [23], into 50- $\mu \mathrm{m}$-wide channels spaced $150 \mu \mathrm{m}$ apart. The structuring method frustrates the extraction of RHEED profiles on the same sample, but lateral RHEED profiles are highly reproducible for samples sequentially grown in the same conditions. Hence, by growing sample $\mathrm{G}$ directly after sample $\mathrm{F}$, the $\mathrm{LaAlO}_{3}$ thickness gradient on their surface should be equal. To suppress the formation of oxygen vacancies, which blur the sharp MIT [24], both samples F and G were annealed for $1 \mathrm{~h}$ in 400 mbar of oxygen at $600{ }^{\circ} \mathrm{C}$ after deposition. This did not affect the measured RHEED profiles [18].

Figure 5 summarizes the effect of a $\mathrm{LaAlO}_{3}$ thickness gradient from four to three unit cells on the conductivity at the $\mathrm{LaAlO}_{3} / \mathrm{SrTiO}_{3}$ interface. Figure 5(b) shows that a transition (a)

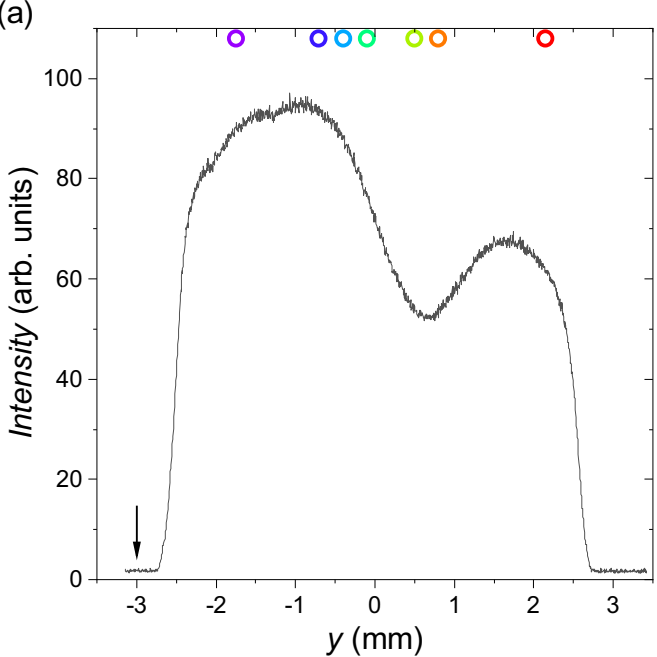

(b)

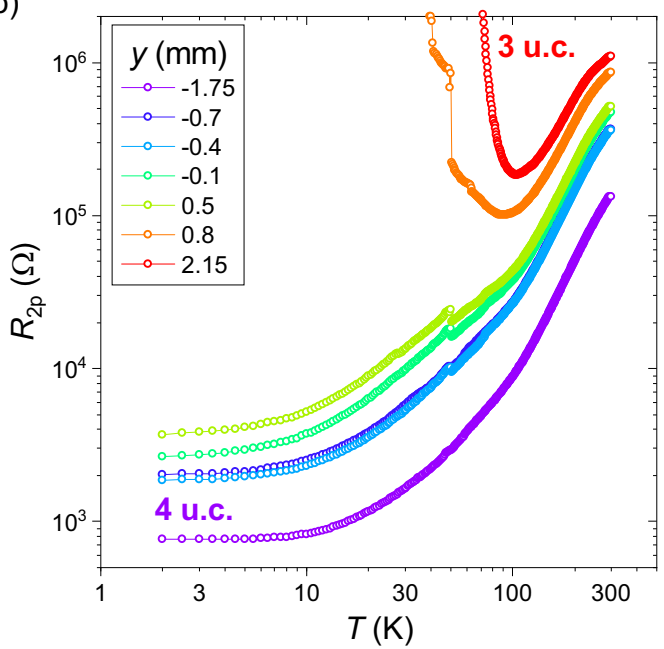

(c)
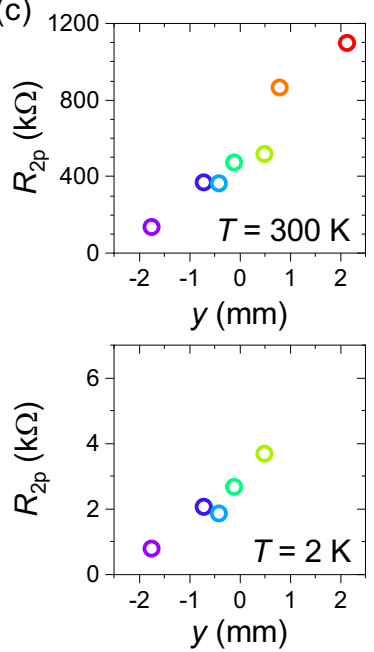

FIG. 5. Metal-insulator transition at the $\mathrm{LaAlO}_{3} / \mathrm{SrTiO}_{3}$ interface along a $\mathrm{LaAlO}_{3}$ thickness gradient of 4 to 3 uc. (a) RHEED intensity versus position on the calibration sample F. The colored circles indicate the positions of the measured channels on sample $\mathrm{G}$, the arrow the plume center position. (b) Two-point resistance $R_{2 \mathrm{p}}$ versus temperature $T$ of measured channels on sample G. (c) $R_{2 \mathrm{p}}$ versus $y$, at $T=300 \mathrm{~K}$ (top panel) and at $T=2 \mathrm{~K}$ (bottom panel). 
from metallic to insulating behavior occurs between $y=0.5$ and $0.8 \mathrm{~mm}$. The positions of these curves on the RHEED profile in Fig. 5(a) suggest that the MIT concurs with the minimum RHEED intensity. In the diffraction interpretation of RHEED oscillations, this minimum corresponds to a toplayer coverage of $\sim 0.5$. Because of the sharpness of the MIT with $\mathrm{LaAlO}_{3}$ layer thickness [7], this implies that half of the interface area is locally conducting, while the other half is insulating. Because the distribution of adatoms during layer-by-layer growth is random, the distribution of conducting and insulating patches is equally irregular. Hence, the "critical" coverage of 0.5 may be interpreted as a percolative threshold, in good agreement with theoretical values for twodimensional square lattices [25]. We note that, despite the reproducibility of profiles across samples grown in the same conditions, the minimum in RHEED intensity may not be in the exact same position on both samples and the actual percolative threshold may differ.

Above the MIT, the channel resistance increases monotonically with distance to the plume center during deposition as shown in Fig. 5(c). In a homogeneous Drude model, this suggests that the carrier mobility, density, or both decrease monotonically with distance to the plume center. Our twoprobe devices do not allow to measure mobility and density separately by, e.g., the Hall effect, but we can estimate the mobility using the residual-resistance ratio, $R R R$, defined as $R(300 \mathrm{~K}) / R(2 \mathrm{~K})$. In the metallic channels, the $R R R$ is not correlated with position and values range from 140 to 193, which would correspond to an electron mobility of $800-1200 \mathrm{~cm}^{2} / \mathrm{Vs}$ at $T=2 \mathrm{~K}$ [26]. We note that the $R R R$ in our devices could be influenced by a temperature-dependent contact resistance, although this should have the same effect in all metallic channels. Hence we conclude that the electron mobility does not significantly vary across these devices and that the effective carrier density decreases as a function of distance to the plume center. This could be mediated either by a monotonically decreasing local free carrier density, or by a similar decline of the total conducting area at the interface. In the later case, the network of conducting regions becomes more weakly interconnected further away from the plume center, eventually leading to a metal-insulator transition at the percolative threshold.

One would expect that such a patchy network of percolating conducting regions is accompanied by 1-u.c.-high islands on the $\mathrm{LaAlO}_{3}$ surface $[27,28]$. If these islands are large enough, they should be observable using scanning-probe techniques. Therefore, we performed an ex situ atomic force microscopy (AFM) study on sample E [18]. Surprisingly, a smooth terrace-and-step structure is observed along the entire thickness gradient, with no indication of the formation of 1-u.c. high islands. As discussed in the Supplemental Material [18], we attribute this to either a too small island size, or masking of the islands by small molecules adsorbing to the polar $\mathrm{LaAlO}_{3}$ surface. Higher resolution AFM or scanning tunneling microscopy (STM) imaging [28] performed in situ could circumvent these problems; this would enable to study directly the relation between RHEED intensity and surface morphology, which despite the widespread use of RHEED for growth monitoring is not yet fully understood [29-31].
Because our ex situ AFM images do not yield direct evidence for a coverage gradient, we consider an alternative possibility for the sharp MIT observed in Fig. 5. Besides $\mathrm{LaAlO}_{3}$ film thickness, the stoichiometry of the $\mathrm{LaAlO}_{3}$ film is critical for the emergence of conductivity at the $\mathrm{LaAlO}_{3} / \mathrm{SrTiO}_{3}$ interface [32-34]. Especially in Ref. [32], a very sharp MIT was observed as a function of the La/Al ratio: films rich in La yielded an insulating interface, where Al-rich $\mathrm{LaAlO}_{3}$ films induced conductivity. Hence if our films also have a lateral variation in stoichiometry, this might underlie the MIT observed here. In PLD, it is well known that the composition of the plasma plume may vary away from its center $[15,16,35]$. However, these studies also show that, if there is a difference at all, heavy elements such as La remain closer to the plume center, where light elements like $\mathrm{Al}$ are more spread out. Therefore, any off-stoichiometry in our films away from the plume center would be Al-rich, which by itself does not yield an insulating interface. Moreover, a large systematic variation in stoichiometry across the film would affect the XRR measurements through the mass density, contrary to our observations [18]. Based on these considerations, we argue that the MIT observed in Fig. 5 is unlikely due to a stoichiometry variation across the sample, but the result of a gradient in nominal $\mathrm{LaAlO}_{3}$ layer thickness from 4 to 3 u.c., as the lateral RHEED profile in Fig. 5(a) implies.

\section{DISCUSSION}

In this study, we focus on the growth of $\mathrm{LaAlO}_{3}$ on $\mathrm{SrTiO}_{3}$ by PLD. But the presented method of measuring post-deposition RHEED profiles is not limited to PLD or to complex oxides. In principle, it can be applied to any film that grows in a layer-by-layer mode, of any material. It therefore opens numerous possibilities to study thickness-dependent phenomena in other systems on single samples. By growing wedge-shaped layers, the behavior of films, interfaces and superlattices can be studied as a function of thickness on a unit-cell scale. Such studies would require local probes rather than, e.g., sample-wide synchrotron beams; our XRR measurements show that this is possible even with laboratory $\mathrm{x}$-ray sources.

We anticipate several applications of lateral RHEED profiles to characterize thin-film growth. These make use of postdeposition analysis as introduced here, or could be based on measurements taken between growth steps or even operando. For the latter, it would be beneficial to scan the electron beam across the sample surface rather than moving the sample through the beam. This improves measurement speed and possibly suppresses noise from mechanical movement, but also poses challenges to keep the diffraction conditions constant throughout the scan. Such operando monitoring or scanning between growth steps could be of particular use for large-area (pulsed laser) deposition. The thus obtained lateral RHEED profiles can be used to adjust the alignment of the material source (in PLD: plasma plume) to the substrate during growth. In principle, this allows to eliminate thickness variations in large-area films with unit-cell precision. On a more fundamental level, lateral RHEED profiles can be valuable for understanding the basic processes of thin-film deposition. For example, the effect of deposition parameters such as process 
pressure, substrate temperature, and substrate-target distance on the distribution of deposited material across the substrate can be quantified. Because the RHEED-profile method is in situ, sensitive on a unit-cell level, and makes use of equipment already present in most deposition chambers, it is an ideally suited tool for such studies.

\section{CONCLUSION}

In summary, we introduced a method to spatially resolve submonolayer thickness variations in epitaxially grown thin films using reflection high-energy electron diffraction. We studied the specific case of $\mathrm{LaAlO}_{3}$ thin films grown on $\mathrm{SrTiO}_{3}$ substrates by pulsed laser deposition, but in principle the method is applicable to any material growing in the layer-by-layer growth mode. As a function of position on the sample, lateral oscillations in the diffraction pattern are observed after the deposition of a thin film of $\mathrm{LaAlO}_{3}$. The oscillation pattern depends on factors such as the relative positioning of the plasma plume to the substrate and the nominal thickness of the grown film. Local $\mathrm{x}$-ray reflectivity measurements confirm the hypothesis that the oscillations are due to a thickness gradient in the film, away from the plume center during deposition. Hence each oscillation represents a full cycle of top-layer coverage and each peak indicates a thickness difference of one unit cell.

The ability to measure in situ atomic-scale thickness variations in epitaxially grown thin films directly after growth, enables straightforward and accurate engineering of these thickness gradients. This paves the way to study thicknessdependent phenomena in a single sample, of which the metalinsulator transition at the $\mathrm{LaAlO}_{3} / \mathrm{SrTiO}_{3}$ interface discussed here is a premier example. Because the equipment required for performing such measurements is already present in most deposition chambers, the method may find widespread application in studying film-growth dynamics as well as in engineering (the elimination of) thickness differences across a large deposition area.

\section{ACKNOWLEDGMENTS}

We thank W. Braun, J. Mannhart, and H. Boschker for valuable discussions and comments on the manuscript. The authors acknowledge financial support through the DESCO program of the Foundation for Fundamental Research on Matter (FOM), associated with the Netherlands Organization for Scientific Research (NWO).
[1] D. D. Fong, G. B. Stephenson, S. K. Streiffer, J. A. Eastman, O. Aucielo, P. H. Fuoss, and C. Thompson, Ferroelectricity in ultrathin perovskite films, Science 304, 1650 (2004).

[2] M. Huijben, L. W. Martin, Y. H. Chu, M. B. Holcomb, P. Yu, G. Rijnders, D. H. A. Blank, and R. Ramesh, Critical thickness and orbital ordering in ultrathin $\mathrm{La}_{0.7} \mathrm{Sr}_{0.3} \mathrm{MnO}_{3}$ films, Phys. Rev. B 78, 094413 (2008).

[3] A. V. Boris, Y. Matiks, E. Benckiser, A. Frano, P. Popovich, V. Hinkov, P. Wochner, M. Castro-Colin, E. Detemple, V. K. Malik, C. Bernhard, T. Prokscha, A. Suter, Z. Salman, E. Morenzoni, G. Cristiani, H. U. Habermeier, and B. Keimer, Dimensionality control of electronic phase transitions in nickeloxide superlattices, Science 332, 937 (2011).

[4] D. J. Groenendijk, C. Autieri, J. Girovsky, M. C. MartinezVelarte, N. Manca, G. Mattoni, A. M. R. V. L. Monteiro, N. Gauquelin, J. Verbeeck, A. F. Otte, M. Gabay, S. Picozzi, and A. D. Caviglia, Spin-Orbit Semimetal $\mathrm{SrIrO}_{3}$ in the Two-Dimensional Limit, Phys. Rev. Lett. 119, 256403 (2017).

[5] T. L. Meyer, L. Jiang, S. Park, T. Egami, and H. N. Lee, Strainrelaxation and critical thickness of epitaxial $\mathrm{La}_{1.85} \mathrm{Sr}_{0.15} \mathrm{CuO}_{4}$ films, APL Mater. 3, 126102 (2015).

[6] H. Y. Hwang, Y. Iwasa, M. Kawasaki, B. Keimer, N. Nagaosa, and Y. Tokura, Emergent phenomena at oxide interfaces, Nat. Mater. 11, 103 (2012).

[7] S. Thiel, G. Hammerl, A. Schmehl, C. W. Schneider, and J. Mannhart, Tunable quasi-two-dimensional electron gases in oxide heterostructures, Science 313, 1942 (2006).

[8] G. Herranz, F. Sánchez, N. Dix, M. Scigaj, and J. Fontcuberta, High mobility conduction at (110) and (111) $\mathrm{LaAlO}_{3} / \mathrm{SrTiO}_{3}$ interfaces, Sci. Rep. 2, 758 (2012).

[9] J. F. Ge, Z. L. Liu, C. Liu, C. L. Gao, D. Qian, Q. K. Xue, Y. Liu, and J. F. Jia, Superconductivity above $100 \mathrm{~K}$ in single-layer FeSe films on doped $\mathrm{SrTiO}_{3}$, Nat. Mater. 14, 285 (2015).
[10] X. R. Wang, C. J. Li, W. M. Lü, T. R. Paudel, D. P. Leusink, M. Hoek, N. Poccia, A. Vailionis, T. Venkatesan, J. M. Coey, E. Y. Tsymbal, Ariando, and H. Hilgenkamp, Imaging and control of ferromagnetism in $\mathrm{LaMnO}_{3} / \mathrm{SrTiO}_{3}$ heterostructures, Science 349, 716 (2015).

[11] J. H. Neave, B. A. Joyce, P. J. Dobson, and N. Norton, Dynamics of film growth of GaAs by MBE from Rheed observations, Appl. Phys. A 31, 1 (1983).

[12] J. M. Van Hove, C. S. Lent, P. R. Pukite, and P. I. Cohen, Damped oscillations in reflection high energy electron diffraction during GaAs MBE, J. Vac. Sci. Technol. B 1, 741 (1983).

[13] J. H. Neave, P. J. Dobson, B. A. Joyce, and J. Zhang, Reflection high-energy electron diffraction oscillations from vicinal surfaces - A new approach to surface diffusion measurements, Appl. Phys. Lett. 47, 100 (1985).

[14] W. Braun, Springer Tracts in Modern Physics, Vol. 154 (Springer Science \& Business Media, New York, 1999), pp. 1-217.

[15] A. Ojeda-G-P, C. W. Schneider, M. Döbeli, T. Lippert, and A. Wokaun, Plasma plume dynamics, rebound, and recoating of the ablation target in pulsed laser deposition, J. Appl. Phys. 121, 135306 (2017).

[16] A. Ojeda-G-P, M. Döbeli, and T. Lippert, Influence of plume properties on thin film composition in pulsed laser deposition, Adv. Mater. Interfaces 5, 1701062 (2018).

[17] G. J. H. M. Rijnders, G. Koster, D. H. A. Blank, and H. Rogalla, In situ monitoring during pulsed laser deposition of complex oxides using reflection high energy electron diffraction under high oxygen pressure, Appl. Phys. Lett. 70, 1888 (1997).

[18] See Supplemental Material at http://link.aps.org/supplemental/ 10.1103/PhysRevMaterials.4.083806 for RHEED patterns during growth, additional XRR data, extraction of thickness variation from RHEED profiles, AFM images along a thickness 
gradient, a sample list, and RHEED profiles for a circular spot and as a function of temperature.

[19] A. Das, S. D. Singh, R. J. Choudhari, S. K. Rai, and T. Ganguli, Data-reduction procedure for correction of geometric factors in the analysis of specular X-ray reflectivity of small samples, J. Appl. Crystallogr. 51, 1295 (2018).

[20] A. Ohtomo and H. Y. Hwang, A high-mobility electron gas at the $\mathrm{LaAlO}_{3} / \mathrm{SrTiO}_{3}$ heterointerface, Nature 427, 423 (2004).

[21] N. C. Bristowe, P. B. Littlewood, and E. Artacho, Surface defects and conduction in polar oxide heterostructures, Phys. Rev. B 83, 205405 (2011).

[22] L. Yu and A. Zunger, A polarity-induced defect mechanism for conductivity and magnetism at polar-nonpolar oxide interfaces, Nat. Commun. 5, 5118 (2014).

[23] N. Banerjee, M. Huijben, G. Koster, and G. Rijnders, Direct patterning of functional interfaces in oxide heterostructures, Appl. Phys. Lett. 100, 41601 (2012).

[24] Z. Q. Liu, L. Sun, Z. Huang, C. J. Li, S. W. Zeng, K. Han, W. M. Lü, T. Venkatesan, and Ariando, Dominant role of oxygen vacancies in electrical properties of unannealed $\mathrm{LaAlO}_{3} / \mathrm{SrTiO}_{3}$ interfaces, J. Appl. Phys. 115, 054303 (2014).

[25] S. Kirkpatrick, Percolation and Conduction, Rev. Mod. Phys. 45, 574 (1973).

[26] Y. Xie, C. Bell, Y. Hikita, S. Harashima, and H. Y. Hwang, Enhancing electron mobility at the $\mathrm{LaAlO}_{3} / \mathrm{SrTiO}_{3}$ interface by surface control, Adv. Mater. 25, 4735 (2013).

[27] M. C. Sullivan, M. J. Ward, A. Gutiérrez-Llorente, E. R. Adler, H. Joress, A. Woll, and J. D. Brock, Complex oxide growth using simultaneous in situ reflection high-energy electron diffraction and $\mathrm{x}$-ray reflectivity: When is one layer complete? Appl. Phys. Lett. 106, 031604 (2015).

[28] S. Gerhold, M. Riva, B. Yildiz, M. Schmid, and U. Diebold, Adjusting island density and morphology of the
$\mathrm{SrTiO}_{3}(110)-(4 \times 1)$ surface: Pulsed laser deposition combined with scanning tunneling microscopy, Surf. Sci. 651, 76 (2016).

[29] S. Clarke and D. D. Vvedensky, Origin of Reflection High-Energy Electron-Diffraction Intensity Oscillations During Molecular-Beam Epitaxy: A Computational Modeling Approach, Phys. Rev. Lett. 58, 2235 (1987).

[30] U. Korte and P. A. Maksym, Role of the Step Density in Reflection High-Energy Electron Diffraction: Questioning the Step Density Model, Phys. Rev. Lett. 78, 2381 (1997).

[31] W. Braun, L. Däweritz, and K. H. Ploog, Origin of Electron Diffraction Oscillations During Crystal Growth, Phys. Rev. Lett. 80, 4935 (1998).

[32] M. P. Warusawithana, C. Richter, J. A. Mundy, P. Roy, J. Ludwig, S. Paetel, T. Heeg, A. A. Pawlicki, L. F. Kourkoutis, M. Zheng, M. Lee, B. Mulcahy, W. Zander, Y. Zhu, J. Schubert, J. N. Eckstein, D. A. Muller, C. S. Hellberg, J. Mannhart, and D. G. Schlom, $\mathrm{LaAlO}_{3}$ stoichiometry is key to electron liquid formation at $\mathrm{LaAlO}_{3} / \mathrm{SrTiO}_{3}$ interfaces, Nat. Commun. 4, 2351 (2013).

[33] E. Breckenfeld, N. Bronn, J. Karthik, A. R. Damodaran, S. Lee, N. Mason, and L. W. Martin, Effect of Growth Induced (Non)Stoichiometry on Interfacial Conductance in $\mathrm{LaAlO}_{3} / \mathrm{SrTiO}_{3}$, Phys. Rev. Lett. 110, 196804 (2013).

[34] H. K. Sato, C. Bell, Y. Hikita, and H. Y. Hwang, Stoichiometry control of the electronic properties of the $\mathrm{LaAlO}_{3} / \mathrm{SrTiO}_{3}$ heterointerface, Appl. Phys. Lett. 102, 251602 (2013).

[35] K. Orsel, R. Groenen, B. Bastiaens, G. Koster, G. Rijnders, and K. J. Boller, Laser-induced fluorescence analysis of plasmas for epitaxial growth of $\mathrm{YBiO}_{3}$ films with pulsed laser deposition, APL Mater. 4, 126102 (2016). 